\section{IDDF2018-ABS-0138 COMPARISON OF THE LONG-TERM OUTCOMES OF ENDOSCOPIC PAPILLARY LARGE-BALLOON DILATION ALONE VERSUS ENDOSCOPIC SPHINCTEROTOMY FOR REMOVAL OF BILE DUCT STONES}

Wenying Yang*. Ruijin Hospital, Affiliated to Shanghai Jiao Tong University School of Medicine, China

\subsection{6/gutjnl-2018-IDDFabstracts.219}

Background Endoscopic papillary large-balloon dilation (EPLBD) is an alternative to endoscopic sphincterotomy (EST) for treatment of common bile duct (CBD) stones. However, limited data exist regarding the comparison of the long-term outcomes for these techniques. In this study, we aimed to compare the long-term outcomes after EST with those after EPLBD alone for removal of CBD stones.

Methods The records of patients with EST or EPLBD alone referred for CBD stones retrieval between June 2008, and July 2015 were retrospectively reviewed. Complete stone clearance, ERCP-related adverse events, and late biliary complications during long-term follow-up were analysed.

Results Basic patient characteristics were similar between the groups that underwent EST $(n=60)$ and EPLBD alone $(n=161)$. EPLBD alone compared with EST resulted in similar outcomes in terms of complete stone clearance $(99.4 \%$ vs $100 \%, \mathrm{p}=0.54)$ and ERCP-related adverse events $(6.8 \%$ vs $10.0 \%, \mathrm{p}=0.41)$. The mean duration of the follow-up was 74.5 months and 71.6 months who underwent EST and EPLBD alone, respectively $(p=0.42)$. Late biliary complications were frequently occurred in the EST group than EPLBD alone group (11 [18.6\%] vs 16 [10.2\%]), although the difference did not reach statistical significance $(p=0.11)$. Furthermore, the incidence of cholangitis without stone recurrence was significantly higher in the EST group than EPLBD alone group (5.1\% vs $0 \%, \mathrm{p}=0.02)$.

Conclusions As an alternative to EST, EPLBD has similar efficacy and safety for managing CBD stones. During longterm follow-up, patients who underwent EPLBD alone may have less late biliary complications compared with those after EST.

\section{IDDF2018-ABS-0144 SINGLE-OPERATOR PERORAL CHOLANGIOSCOPY IN THE TREATMENT OF DIFFICULT BILIARY STONES: A SYSTEMATIC REVIEW AND META-ANALYSIS}

${ }^{1}$ Zheng Jin*, ${ }^{2}$ Xiaofeng Zhang. 'Hangzhou Geriatric Hospital, Hangzhou First People's Hospital Group, Hangzhou, China; ${ }^{2}$ Department of Gastroenterology, Hangzhou First People's Hospital, Nanjing Medical University, Hangzhou, China

\subsection{6/gutjnl-2018-IDDFabstracts.220}

Background Current evidence supporting the utility of singleoperator peroral cholangioscope (SOPOC) in the management of difficult bile duct stones is limited. We conducted the present systemic review and meta-analysis to evaluate the efficacy and safety of SOPOC in the treatment of difficult bile duct stones.

Methods A search of studies up to January 2018 was acquired, using MEDLINE, EMBASE, the Cochrane Library and Google Scholar. Quality assessment of the studies was completed with a modified Newcastle-Ottawa Scale. The main outcomes of interest were single-session stone clearance rate, complete stone clearance rate and adverse events. We calculated the pooled proportions with random-effects models. Subgroup analyses were also performed based on SOPOC type.

Results A total of 21 studies involving 2490 patients met the inclusion criteria. Average number of stones per patient was 2.2. Mean stone size was $17.4 \mathrm{~mm}$. The pooled proportion of patients with single-session stone clearance was $72.5 \% \quad(95 \%$ confidence interval [95\% CI], 63.2\%-80.9\%). (Figure 1). Complete stone clearance was achieved in 94.7\% (95\% CI, 90.1\%-98.1\%) of patients with median endoscopic sessions of 2.0. The pooled adverse event rate was 5.5\% (95\% CI, $3.3 \%-8.2 \%)$. For SOPOC type SpyGlass and SpyGlass DS, single-session stone clearance rate was 69.1\% (95\%CI: $56.3 \%$ $80.6 \%)$ and $80.0 \%$ (95\% CI: $75.8 \%-83.9 \%)$, respectively. Complete stone clearance rate was $92.8 \%$ (95\%CI: $86.5 \%$ 97.5\%) and 96.7\% (95\%CI: 88.1\%-100.0\%), respectively. Adverse events rate was 6.2\% (95\%CI: $3.4 \%-9.7 \%)$ and $3.0 \%$ (95\% CI: $1.8 \%-4.4 \%)$, respectively.

Conclusions SOPOC is an effective and safe management option for the treatment of bile duct stones when conventional methods have failed. More randomised controlled trials 


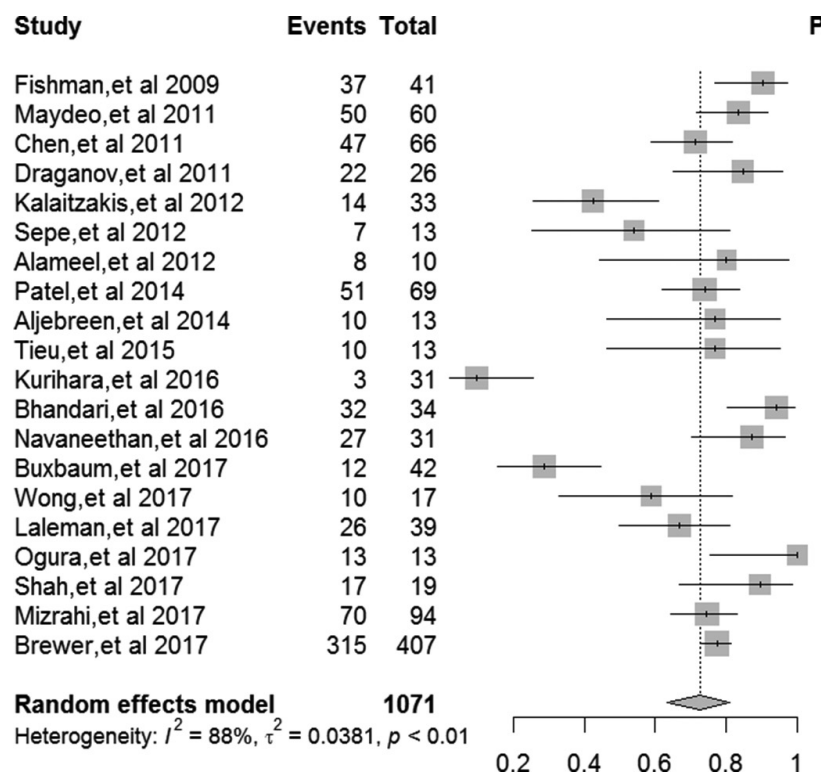

Proportion $\quad 95 \%-\mathrm{Cl}$ Weight

$0.902[0.769 ; 0.973] \quad 5.3 \%$

$0.833[0.715 ; 0.917] \quad 5.6 \%$

$0.712[0.587 ; 0.817] \quad 5.6 \%$

$0.846[0.651 ; 0.956] \quad 5.0 \%$

$0.424[0.255 ; 0.608] \quad 5.2 \%$

$0.538[0.251 ; 0.808] \quad 4.2 \%$

$0.800[0.444 ; 0.975] \quad 3.8 \%$

$0.739[0.619 ; 0.837] \quad 5.7 \%$

$0.769[0.462 ; 0.950] \quad 4.2 \%$

$0.769[0.462 ; 0.950] \quad 4.2 \%$

$0.097[0.020 ; 0.258] \quad 5.1 \%$

$0.941[0.803 ; 0.993] \quad 5.2 \%$

$0.871[0.702 ; 0.964] \quad 5.1 \%$

$0.286[0.157 ; 0.446] \quad 5.4 \%$

$0.588[0.329 ; 0.816] \quad 4.5 \%$

$0.667[0.498 ; 0.809] \quad 5.3 \%$

$1.000[0.753 ; 1.000] \quad 4.2 \%$

$0.895[0.669 ; 0.987] \quad 4.6 \%$

$0.745[0.644 ; 0.829] \quad 5.8 \%$

$0.774[0.730 ; 0.814] \quad 6.1 \%$

$0.725[0.632 ; 0.809] 100.0 \%$

\section{Abstract IDDF2018-ABS-0144 Figure 1}

are required to elucidate the precise role of SOPOC during ERCP.

\section{IDDF2018-ABS-0147 EFFICACY AND SAFETY OF ENDOSCOPIC RETROGRADE CHOLANGIOPANCREATOGRA- PHY IN NONAGENARIANS: A COMPARATIVE STUDY}

'Zheng Jin*, 'Xiaofeng Zhang. 'Hangzhou Geriatric Hospital, Hangzhou First People's Hospital Group, Hangzhou, China; ${ }^{2}$ Department of Gastroenterology, Hangzhou First People's Hospital, Nanjing Medical University, Hangzhou, China

\subsection{6/gutjnl-2018-IDDFabstracts.221}

Background Little information is available on the long-term outcome of endoscopic retrograde cholangiopancreatography (ERCP) for pancreatobiliary diseases in nonagenarians $(\geq 90$ years). This study was to evaluate the efficacy and the safety of of ERCP in the treatment of patients 90 years of age and older.

Methods Consecutive nonagenarian patients who underwent therapeutic ERCP between May 2005 and January 2017 at a tertiary-care centre were retrospectively reviewed. One hundred and fifty-five patients aged 90 years and older were identified (Group A). Matched by gender and ratio 1:2, 310 patients under 65 years were assigned to control group (Group B). Clinical features, endoscopic findings, interventions, long-term results of ERCP for a mean follow-up of 12 months were assessed and compared between the two groups. Results Group A had a higher incidence of concomitant diseases, acute cholangitis, and acute cholecystitis, as compared to group B $(\mathrm{p}<0.05)$. There was no significant difference in the technical success rate and procedure time between the two groups. The rate of post-ERCP pancreatitis was significantly less in the group A compared to the group $\mathrm{B}(\mathrm{p}<0.05)$. The occurrence of haemorrhage, perforation, and other complications was not statistically different among the two groups. The mortality directly related to the ERCP procedure was zero.

Conclusions ERCP is safe and effective for the management of pancreatobiliary diseases in patients aged 90 years or older.

\section{IDDF2018-ABS-0150 RISK OF HEPATOCELLULAR CANCER AND DEATH/TRANSPLANT BETWEEN INACTIVE HEPATITIS B AND HBEAG-NEGATIVE CHRONIC HEPATITIS B WITH ANTIVIRAL AGENTS}

${ }^{1}$ Gwang Hyeon Choi* ${ }^{1}$ Gi-Ae Kim, ${ }^{1}$ Jonggi Choi, ${ }^{2}$ Seungbong Han, ${ }^{1}$ Young-Suk Lim. ${ }^{1}$ Asan Medical Center, Korea, South; ${ }^{2}$ Gachon University, Korea, South

\subsection{6/gutjnl-2018-IDDFabstracts.222}

Background Nucleos[t]ide analogues (NUCs) treatment in HBenegative chronic hepatitis $\mathrm{B}(\mathrm{CHB})$ patients would maintain a similar virologic and biochemical state compared to inactive hepatitis. And, sustained reduction of hepatitis B viral load by NUCs is helpful in lowering the risk of HCC. The purpose of this study was to evaluate the cumulative incidence of hepatocellular carcinoma (HCC) and death/transplantation in patients with inactive patients compared with $\mathrm{HBeAg}$-negative $\mathrm{CHB}$ patients treated with NUCs.

Methods We performed a retrospective analysis of data from 3202 consecutive adult patients with non-cirrhotic $\mathrm{CHB}$, inactive hepatitis $(n=2,677)$ and $\mathrm{HBeAg}$-negative hepatitis treated with NUCs between January 2000 and December 2013. Data were collected from patients for median 5.4 years and analysed by a multivariable Cox proportional hazards model for the entire cohort and propensity score-matched cohort.

Results During the study period, 127 (4.0\%) developed HCC, $156(4.8 \%)$ died or received transplantation. The annual risk of developing HCC and death/transplantation of inactive 